\title{
Tribute to John Walter Clark on his 60th birthday
}

\section{Document Version}

Accepted author manuscript

Link to publication record in Manchester Research Explorer

\section{Citation for published version (APA):}

Bishop, RF., Campbell, CE., Gernoth, KA., Krotscheck, E., \& Ludeña, EV. (1998). Tribute to John Walter Clark on his 60 th birthday. In J. da Providência, \& FB. Malik (Eds.), Condensed Matter Theories, Vol. 13 (pp. 1-20). Nova Science Publishers. http://personalpages.manchester.ac.uk/staff/raymond.bishop/RFB_papers/[144] CMT_13(1998)1

\section{Published in:}

Condensed Matter Theories, Vol. 13

\section{Citing this paper}

Please note that where the full-text provided on Manchester Research Explorer is the Author Accepted Manuscript or Proof version this may differ from the final Published version. If citing, it is advised that you check and use the publisher's definitive version.

\section{General rights}

Copyright and moral rights for the publications made accessible in the Research Explorer are retained by the authors and/or other copyright owners and it is a condition of accessing publications that users recognise and abide by the legal requirements associated with these rights.

\section{Takedown policy}

If you believe that this document breaches copyright please refer to the University of Manchester's Takedown Procedures [http://man.ac.uk/04Y6Bo] or contact uml.scholarlycommunications@manchester.ac.uk providing relevant details, so we can investigate your claim.

\section{OPEN ACCESS}




\title{
CONDENSED MATTER THEORIES
}

\author{
VOLUME 13
}

Edited by

João da Providência

Universidad de Coimbra

F.B. Malik

University of Southern Illinois at Carbondale

Nova Science Publishers, Inc. - Commack, New York 


\section{CONDENSED MATTER THEORIES 21th International Workshop on Condensed Matter Theories Luso, Portugal 22 - 26 September, 1997}

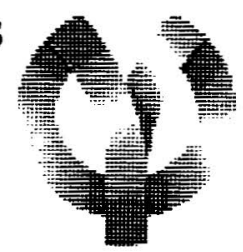

Editorial Production: Susan Boriotti Office Manager: Annette Hellinger Graphics: Frank Grucci and John TLustachowski Information Editor: Tatiana Shohov Book Production: Donna Dennis, Patrick Davin, Christine Mathosian, Tammy Sauter and Diane Sharp

Circulation: Maryanne Schmidt Marketing/Sales: Cathy DeGregory

Library of Congress Cataloging-in Publication Data available upon request

ISBN 1-56072-602-4 ISSN 0893-861X

Copyright 1998 Nova Science Publishers, Inc. 6080 Jericho Turnpike, Suite 207 Commack, New York 11725 Tele. 516-499-3103 Fax 516-499-3146

E Mail: Novascience@earthlink.net Novascil@aol.com

Web Site: http://www.nexusworld.com/nova

All rights reserved. No part of this book may be reproduced, stored in a retrieval system or transmitted in any form or by any means: electronic, electrostatic, magnetic, tape, mechanical, photocopying, recording or otherwise without permission from the publishers.

Printed in the United States of America 


\title{
TRIBUTE TO JOHN WALTER CLARK ON HIS 60TH BIRTHDAY
}

\author{
R. F. Bishop \\ Department of Physics \\ University of Manchester Institute of Science and Technology, \\ Manchester, M60 1QD, UNITED KINGDOM \\ C. E. Campbell \\ School of Physics and Astronomy \\ University of Minnesota, Minneapolis, MN 55455, USA \\ K. A. Gernoth \\ Institut für Theoretische Physik \\ Universität zu Köln, D-50937 Köln, GERMANY \\ E. Krotscheck \\ Institut für Theoretische Physik \\ Johannes-Kepler-Unniversität, A-4040 Linz-Auhof, AUSTRIA

\section{E. V. Ludeña} \\ Centro de Quimica \\ Instituto Venezolano de Investigaciones Cientificas, Caracas 1020-A, VENEZUELA
}

One of the highlights of the XIXth International Workshop on Condensed Matter Theories was the special session on Saturday, June 17, 1995, dedicated to honor John Walter Clark on the occasion of his 60th birthday.

This workshop series owes a great deal to John Clark for the leadership, enthusiasm, and perseverance he has shown in the organization of these scientific events. In fact, his participation as a member of the International Advisory Board, the Editorial Board, and the Workshop Trust Fund Committee has been fundamental in maintaining the continuity and the high scientific standards of these workshops. For this reason, the organizing committee of the XIXth International Workshop on Condensed Matter Theories saw it fit to render this well-deserved tribute to John Clark 
by dedicating one of the sessions to him.

But in addition, there are two other aspects that we considered when making this decision. The first was the wish to express our respect and admiration to John Clark as an outstanding scientist. The second, our desire to pay our tribute to John Clark as an enlightened and generous human being.

This workshop afforded the participants the opportunity to honor John Clark. The workshop was felt to be a particularly appropriate setting for this celebration, since Clark has been instrumental in developing and organizing this workshop series from its inception. Many collaborations which have fostered the advancement of condensed matter theories and the sustenance of state of the art research in developing countries have been a direct result of Clark's tireless efforts in these directions.

As a scientist, John Clark has left his mark of high productivity and originality in diverse areas of physics such as quantum mechanics of many-body systems, quantum fluids, nuclear structure and dynamics, dense matter astrophysics, quantum control theory, and theoretical biophysics. He has authored over 180 articles published in professional journals, books, and conference proceedings.

John Walter Clark was born in Lockhart, Texas, USA, on April 7, 1935. He did his undergraduate work at the University of Texas at Austin, where he obtained his B.S. degree in 1955 and his M.A. degree in 1957. From there he moved to Washington University in St. Louis, where he received his Ph.D. degree in physics in 1959. In the course of his academic career he was a research associate in physics at Washington University in 1959, a NSF postdoctoral fellow at Princeton University from 1959 to 1962 , an associate research scientist at the Martin Company, Denver, in the summer and fall of 1961, a NATO postdoctoral fellow at the University of Birmingham and at CENS, Saclay; France, from 1962 to 1963, assistant and later associate professor of physics at Washington University, from 1963 to 1972, and full professor of physics at Washington University since 1972.

His professional honors and awards include an Alfred P. Sloan Foundation fellowship from 1965 to 1967 , a NATO senior fellowship in the Laboratorio di Cibernetica, Naples, Italy, in 1972, and the Eugene Feenberg medal for many-body physics in 1987. He is a Fellow of the American Physical Society.

Of the committees on which he has served, we would like to list the International Advisory Committee for the International Workshops on Condensed Matter Theories, the Program Committee for the Conference on Computer Simulation in Brain Science, the International Advisory Board and Program Committee for the Conferences on Recent Progress in Many-Body Theories, the Program Committee for the Conference on Models of Brain Function, and the Program Committee of the Danish Royal Academy's Symposium on Brain and Mind. He has also acted as co-director of the Workshop on Complex Dynamics of Neural Networks.

John Clark arrived in St. Louis in 1957 to do his Ph.D. at Washington University. He became Eugene Feenberg's first student in the essentially new field of many-body physics and much of what Clark has achieved subsequently in this field can be seen to have its origins in this initial period of interaction with Feenberg.

Clark's research on microscopic many-body theory of strongly correlated quantum systems was recognized by the award of the prestigious Eugene Feenberg Memorial Medal in Many-Body Physics to him at the Fifth International Conference on Recent Progress in Many-Body Theories, in Oulu, Finland, in August 1987. A com- 
prehensive overview of this part of Clark's work was presented by one of us (RFB) at that meeting, and is printed in the proceedings (Recent Progress in Many-Body Theories, Vol. 1, A. J. Kallio, E. Pajanne, and R. F. Bishop, eds., Plenum, New York, 1988). We have directly excerpted parts of that article for our overview of this phase of his work. In addition, we present an overview of Clark's work on neural networks and related areas.

Much of Clark's research in many-body physics has been concerned with methods for the quantitative prediction of the ground states and elementary excitations of strongly interacting quantum many-body systems from a completely microscopic starting point. The systems of interest to him have ranged from the helium liquids to finite nuclei, nuclear matter, and neutron star matter. These systems are characterized by having the basic interactions between their constituents so strong that an accurate description in terms of independent-particle models or by means of ordinary perturbation theory is precluded from the outset. Clark is especially known for his contributions over many years to the development and application of the method of correlated basis functions (CBF), which has proven itself to be one of the most effective and viable procedures for dealing with this most important class of quantum many-body systems.

Feenberg and Clark proposed in Clark's first, and their only joint, publication [1] a theory which later became known as the CBF method. Clark's Ph.D. thesis (1959) contains a more detailed development of the method, which includes the first formulation of correlated-basis perturbation theory as a means for the systematic improvement on such single-pass variational treatments as that of Jastrow. At this early date formal prescriptions were also presented for the construction of both offdiagonal and diagonal CBF matrix elements.

The essential steps that paved the way for later practical realizations of the CBF approach to many-fermion systems were outlined in an important early work with Westhaus [6]. Procedures were given there both for the evaluation of off-diagonal CBF matrix elements by cluster expansion techniques and for the transformation to an orthonormal correlated basis. It was also in this paper that the by now familiar Clark-Westhaus form for matrix elements of the kinetic energy operator made its first appearance. The relationship of the CBF method to the quasiparticle picture of Landau was also first explored at this time. With the passage of time one can now see very clearly that many of the key ingredients in the CBF program that is still being carried out today were identified in the early paper by Clark and Westhaus [6]. The collaboration with Westhaus continued and soon led them to consider the formal development of cluster-expansion techniques $[4,6]$, including factorized, or multiplicative, versions of the Iwamoto-Yamada and Aviles-Hartogh-Tolhoek expansions. These techniques have proven to be extremely useful for the subsequent treatment of non-uniform systems such as finite nuclei. Clark returned several years later to work on formal cluster theory, this time in collaboration with Ristig. This work, discussed in more detail below, may nowadays be seen to have provided a fir$\mathrm{m}$ basis for the later development of the highly important techniques of the Fermi hypernetted chain (FHNC) method, which have been so successful in a wide variety of applications to strongly correlated many-body systems and which have played a vital role in a full implementation of the CBF program.

While others largely worked with such quantum fluids as the helium liquids 
and Coulombic systems, Clark originally concentrated on applications in nuclear physics. In a subsequent publication [9], which was to be both the first application of CBF perturbation theory to a Fermi system and the first CBF application to a nuclear problem, it was demonstrated, that the tensor force also produces a strong enhancement in the dipole sum rule.

With the benefit of hindsight however, there is no doubt, that the most important contribution of Clark in this early work on nuclear physics was a seminal paper in 1969 with Bäckman and Chakalakal [12], which initiated a quantitative comparison of the Brueckner and Jastrow approaches for quasi-realistic models of nuclear matter. Taken together with later work on the same subject by Clark and his collaborators this avenue of research clearly pointed to the inadequacy of Brueckner theory for nuclear physics applications as it was then commonly practiced.

One can clearly see now however, that it was the early 1969 paper [12] that set the stage for what was only considerably later to become recognized as the by now well-known "crisis in nuclear matter theory." Briefly stated, the results of Clark and his collaborators showed that the expectation value of the Hamiltonian in a trial wavefunction of Jastrow form could be appreciably lower than the corresponding result using Brueckner theory (or, more precisely, what would nowadays be called lowest-order Brueckner theory, LOBT) and the same quasi-realistic Hamiltonian. The energy variational principle then led inexorably to the conclusion that the Brueckner estimate had to be badly wrong, provided that it was accepted that the variational expectation value in the trial Jastrow state had been accurately evaluated by the cluster expansion techniques then employed by Clark and his coworkers.

At that time however, the nuclear theory community was not yet ready to be convinced by these results. This crisis was widely seen to have become settled by about three years later in favor of the variation and CBF treatments and against the LOBT as performed up to the time of the Bethe review. Looking back, it can be seen, that the 1969 paper of Clark et al. [12] had already sounded the death-knell for LOBT, although few recognized it then.

Clearly the crisis in nuclear matter theory has been resolved. Clark himself gave an update [71] on that crisis in 1978 as a summary of the First International Conference on Recent Progress in Many-Body Theories held in Trieste, that still essentially holds today. The major part of the credit for bringing the results of the confrontation between perturbative (LOBT) and variational (CBF) theories applied to nuclear matter to this resolution undoubtedly belongs to Clark. Both the perturbative and variational schools owe him much for drawing the crisis to their attention and also for playing a large role in its resolution. A very beneficial outcome of the confrontation between CBF and LOBT was the subsequent wide appreciation of the enormous power of the variational and CBF techniques. The subsequent emphasis placed on the complementary roles played by the variational and perturbative approaches has also played a vital role in many later developments. Indeed, the exploration of interconnections between the two approaches is nowadays seen to be at least as important as separate advances in either of the methods.

To return to the CBF method and its development, Clark and his collaborators were instrumental in two particular advances that have proven to be essential for later applications of the method to strongly correlated systems of physical interest. Firstly, for realistic nuclear systems for example, it is crucial to incorporate the large state- 
dependence of the correlations that arises in great part from such strong non-central components in the interaction as the tensor force. There are two distinct ways of including the effects of such correlations in the CBF framework, and both have been developed by Clark and his coworkers. The first alternative is to incorporate the state-dependence into the ground-state trial wavefunction by suitably generalizing the Jastrow form, so that the correlation operator generating the trial states includes state-dependent, especially tensor, terms. Clark, together with Ristig and Ter Louw, gave both the first such systematic procedure for incorporating state-dependent correlations in many-fermion systems and did also the first calculations of nuclear matter with such correlations included $[22,28]$. The second alternative is to work with CBF perturbation theory on top of the usual Jastrow-Slater (non-state-dependent) correlated basis and to include the effects of the state-dependence by going to higher (i.e., at least to second-order) corrections in this perturbative basis. Clark and his collaborators also pursued this approach, although initially only for central potentials. Both of these alternative approaches were later combined, again with the essential collaboration of Ristig, into a powerful and versatile tool for systems such as realistic nuclear matter, to which it was applied. These CBF approaches have later been further developed and refined by others, particularly by the group of Pandharipande, but the groundwork was done in each case by the Clark-Ristig group.

The second fundamental advance in the CBF method that was vital for its adaptation into such a powerful tool concerns the practical evaluation of such CBF matrix elements as the energy expectation value. We have already noted how Clark was involved in the development of cluster-expansion techniques and cluster formalisms suited to this task [4,6]. The later work with Ristig along these lines also revealed some of the inter-connections between the variational and Brueckner techniques. Most importantly however, this formal cluster theory work provided a basis for much of the later diagrammatic analyses of expectation values in the CBF correlated basis $[19,35,37-39]$ and particularly for the extremely important equations of the FHNC type, which perform a resummation of certain classes of cluster contributions to all orders. The development of the FHNC and related cluster resummation techniques was the second crucial step that was necessary for a full quantitative implementation of the CBF program to such strongly correlated systems as the helium liquids, where it has proven so successful. Clark was not directly involved in the invention of FHNC techniques for the evaluation of expectation values, but he recognized their importance immediately and himself has used them and contributed to their further development ever since. He also published in 1979 what must be considered to be the standard review [64] on the variational theory of nuclear matter. This review surveyed in depth both the formal and practical aspects of FHNC methods and established the notation and terminology used thereafter by the practitioners in this field.

Another area in which Clark has made important contributions is the extension and application of the variational and CBF schemes to deal with superfluid systems. One particular system of continued interest has been neutron star matter, whose period of interest to the nuclear many-body community dates from the discovery around 1968-69 that pulsars are neutron stars. Clark first showed, with Chao, that despite some earlier speculations to the contrary, neutron star matter could not be ferromagnetic, but was likely to be superfluid at certain densities [15]. He continued 
this work with his students, carrying out the first serious microscopic investigations of the energy gap and isotropic pairing in neutron star matter within correlated BCS theory and considering both proton superconductivity and neutron matter superfluidity. These results have been used repeatedly ever since as input data for models of glitch phenomena and cooling in neutron stars. Clark has also contributed on both sides in the rather heated debate about the possibility of solidification (crystallization) of the cores of neutron stars. His interest in neutron star matter remains strong; he recently published [161] a realistic CBF investigation of neutron pairing, which has quantitatively verified the earlier prediction that the polarization of the medium produces a substantial suppression of the superfluid gap.

The realization that pulsars are neutron stars, which dates the origin of interest of the many-body community in neutron star matter, occurred almost simultaneously with the appearance of the first signals from Clark's works of the impending "crisis in nuclear matter theory." It is perhaps worth recalling that the excitement and diversion caused by the surge of interest in neutron star matter enabled such manybody theorists as Clark to test and hone their methods on a new problem. Upon subsequently returning to the nuclear matter problem, the crisis found a speedy resolution.

Another noteworthy contribution with Ristig concerns the formulation of a variational theory of the momentum distribution and one-body density matrix for quantum fluids $[50,52,55,60]$. Fundamental structural relations in terms of classes of cluster diagrams were discovered during this development of a cluster theory for the momentum distribution corresponding to a Jastrow trial wavefunction. These relations were later exploited by Fantoni in a derivation of FHNC equations for this function. The Clark-Ristig group subsequently applied their techniques to helium liquids and nuclear matter. Their helium results are well known by the inelastic neutron scattering community, particularly by those involved in the experimental determination of momentum distributions.

The period since about 1979 has seen the further extension of the CBF approach towards providing a comprehensive description of the dynamics and statics of strongly correlated quantum many-body systems. Two formal advances that Clark has achieved with his students and with Krotscheck as his primary collaborator are particularly noteworthy. These are the so-called FHNC' theory and the correlated random-phase approximation (CRPA). In the context of the former problem setting, Krotscheck and Clark extended the FHNC theory for the evaluation of diagonal CBF matrix elements in a Jastrow-Slater correlated basis to the corresponding FHNC' theory for off-diagonal elements. This work led to the definition of the CBF effective interaction and to many illuminating connections with conventional diagrammatic many-body perturbation theory. Exploration of these connections has allowed powerful techniques from ordinary many-body theory, as developed for weakly interacting systems, to be taken over for comparable application to strongly interacting systems within the CBF program. Such familiar quantities as self-energies and quasiparticle interactions have thus been brought under the CBF mantle.

The second major advance has seen the use of CBF theory by Clark and his collaborators to extend the random-phase approximation to the microscopic description of linear response and elementary excitations in strongly interacting systems [84]. This has been achieved by a generalization of time-dependent Hartree-Fock theory 
to a correlated basis. It permitted a genuine microscopic treatment of the elementary excitations in such systems to be made for the first time. Numerous successful applications of this CRPA method have already been made to various systems. Examples include normal unpolarized liquid ${ }^{3} \mathrm{He}$, polarized liquid ${ }^{3} \mathrm{He}$, the electron gas, and closed-shell nuclei.

While Clark and his collaborators have continued the formal development of CBF methods, they have also been at the forefront of applications to real physical systems. A major theme of more recent work has been the application of both CBF perturbation theory and other variational approaches to the quantitative description of the ground states of unpolarized and polarized liquid ${ }^{3} \mathrm{He}$ and of several species of electron-spin-aligned bulk atomic deuterium. Particularly worthy of mention are the variational Monte Carlo calculations with Panoff and others on the ground-state phases of polarized deuterium species. Looking finally to nuclear applications, Mead and Clark [75] gave the first non-trivial application of CBF theory, in the form of a correlated Tamm-Dancoff approximation, to a finite nucleus.

In addition to his mainstream work in many-body theory, Clark has devoted substantial research efforts to quantum control theory (see, e.g., Ref. 183) and to the theory of neural networks. He has made such substantial contributions to both areas that one feels it is only a matter of time before a full CBF-like program flowers to envelop these new enterprises as well. Indeed, particularly in the latter case of theoretical neurobiology, Clark may well be in at the birth of another new field of vast future importance - in much the same way as his own career began nearly forty years ago under his friend and mentor Feenberg, who was then similarly turning his attention to assist at the birth of quantum many-body theory. The wheel then will have turned full circle, and in so doing will surely provide a fitting tribute to the lasting power of the example and guiding light provided by Eugene Feenberg.

Clark's work in the area of neural networks covers an impressive variety of different topics ranging from modeling real, living nervous systems in physiological contexts to applications of artificial neural networks as novel computing devices to fundamental problems in basic science. In addition to his numerous original contributions to this exciting field at the forefront of contemporary science Clark authored several major review articles, providing not only comprehensive accounts of the present state of neural networks research but also excellent introductory reading for the uninitiated.

Of Clark's research in the area of biological neural nets we would like to highlight his work on the mammalian olfactory bulb $[137,149]$, which is considered to be one of the simplest and thus most fundamental of cortical systems in vertebrates, a sort of "hydrogen-atom" of cortical neuronal assemblies. In this work, analog neural network models operating in continuous time, with the activities of the neuronal units representing firing frequencies, have been adopted to describe the basic six-neuron circuit of the olfactory system. Extensive computer simulations of the dynamical behavior of this system revealed the occurrence of sustained, stable oscillations in response to external stimuli, with periods that are typical of the frequencies found in EEG experiments, thus reproducing theoretically one of the most salient features of observed cortical activity. In these studies similar behavior was found also for physiologically more realistic chains and rings of basic olfactory bulb circuits.

Related to these explorations of the olfactory system are Clark's studies $[121,122]$ of cyclic behavior in quasi-randomly connected networks of binary neurons operating 
synchronously in discrete time - work motivated by the neuro-physiological significance of cycles of short periods in living nervous systems. In these papers Clark and collaborators performed extensive empirical studies, by computer simulations, of cycling in such deterministic, finite-state, sequential machines of neuronal units. Accessibility of cycles from initial conditions, periods of cycles, stabilities of cyclic solutions of the neuro-dynamical equations with respect to disturbances by singleneuron state flips, and the average number of cyclic modes have been investigated in detail for networks varying in size, density of interneuronal connections, fraction of inhibitory units, and magnitudes of non-zero neuron-neuron couplings.

Clark's work $[110,116,123]$ on deterministic chaos in quasi-random, continuoustime neural network models with continuous activity variables evolved out of these studies of cyclic behavior in discrete-time, binary networks. Clark and his coinvestigators examined the occurrence of chaotic solutions of the non-linear neurodynamical equations, governing the time-dependence of the analog activities of the neuronal units, in dependence on connectivity, network size, and interneuronal coupling strengths, with the strength of the external stimulus to a net serving as control parameter. For certain ranges of the external input, chaos was found to be quite common in sufficiently large nets, a finding which bears potential biological implications such as the possibility for nervous systems to break out of rigid, stereotyped behavior.

Clark's more recent involvement in neural networks research has focussed mainly on scientific applications of artificial neural networks with an emphasis on problems of current interest in nuclear physics. Layered networks consisting of analog units with feedforward neuron-neuron connections of adjustable strength have been trained with standard backpropagation or backpropagation-like learning algorithms to fit and predict the systematics of the nuclear chart. This work led to remarkably accurate neural-network models of the stability-instability dichotomy of nuclides [154], of nuclidic spins [159], and of proton and neutron binding energies in nuclides $[151,152,155]$ and ultimately culminated in global neural-network models of atomic mass excesses [159] and of branching ratios in decay of unstable nuclides [171]. The latter neural-network models in particular have been found to be superior to conventional, semi-phenomenological, global models of nuclear stability and decay. Apart from the significance of these results for nuclear physics, neural-network modeling of nuclear decay furnished a nice opportunity for exploring, from a more technical point of view, in a real-world application a modified backpropagation algorithm, based on relative entropy [145] as cost function, for teaching networks to predict probabilities [171]. In a similar vein, Gernoth and Clark proposed a modified backpropagation learning scheme designed to train neural networks on data with error bars [175]; this algorithm was tested numerically with success in the context of neural-network modeling of atomic mass excesses.

Clark's reviews on neural networks are notable for their thorough treatment of a vast number of highly relevant aspects of the physics of these non-linear complex systems. His reviews furnish outstanding introductory material to the most common of mathematical models of living nervous systems and their functioning, such as possible biological mechanisms of learning through neural plasticity, and to the basic elements of neuronal anatomy and physiology underlying these models $[108,136,149]$. Moreover, Clark's review articles cover the equilibrium and non-equilibrium statis- 
tical mechanics of deterministic and of probabilistic neural networks $[119,128]$ with deep insight.

We would like to conclude this brief survey of Clark's many activities in the area of neural networks by mentioning, that his reviews also provide a summary of the learning algorithms used in training layered, feedforward artificial neural networks and comprise a discussion of several remarkable applications of neural nets to pressing problems in basic science [160].

Parts of this article have been adapted from the presentation (by R. F. Bishop) of the second Eugene Feenberg Memorial Medal in Many-Body Physics to John Walter Clark at the Fifth International Conference on Recent Progress in Many-Body Theories in Oulu, Finland, in 1987.

\section{JOHN WALTER CLARK PUBLICATIONS}

1. J. W. Clark and E. Feenberg (1959) Simplified treatment for strong short-range repulsions in $N$-particle systems. Phys. Rev. 113, 388-399.

2. J. W. Clark (1960) Effective spin-orbit potential in correlated heavy nuclei. $A n$ nals of Physics 11, 483-500.

3. J. W. Clark (1961) The role of repulsive cores in the photonuclear effect. Can. J. Phys. 39, 385-392.

4. J. W. Clark, D. Chakkalakal, and P. Westhaus (1965) Simple derivation of cluster expansions for many-fermion systems. Progr. Theor. Phys. 34, 726-733.

5. T.-P. Wang and J. W. Clark (1965) Dynamical correlations and the nuclear photoeffect. Progr. Theor. Phys. 34, 776-813.

6. J. W. Clark and P. Westhaus (1966) Method of correlated basis functions. Phys. Rev. 141, 833-857; 149, 990.

7. J. W. Clark and T.-P. Wang (1966) Theory of $\alpha$ matter. Annals of Physics 40, 127-152.

8. P. Westhaus and J. W. Clark (1966) $\Lambda$ binding to nuclear matter. Phys. Lett. 23, 109-111.

9. J. W. Clark and P. Westhaus (1968) Cluster expansions in many-fermion theory, I. Factor-cluster formalisms. J. Math. Phys. 9, 131-148.

10. P. Westhaus and J. W. Clark (1968) Cluster expansions in many-fermion theory, II. Rearrangements of primitive decomposition equations. J. Math. Phys. 9, 149154.

11. G. Mueller and J. W. Clark (1968) The problem of lambda-particle binding in nuclear matter. Nucl. Phys. B7, 227-249.

12. S.-O. Bäckman, D. A. Chakkalakal, and J. W. Clark (1969) Numerical comparison of reaction-matrix and Jastrow methods for nuclear matter. Nucl. Phys. A130, 635-649.

13. L. Rickertsen, B. Block, J. W. Clark, and F. B. Malik (1969) Nuclear heavyion-heavy-ion collisions and the intermediate-state model. Phys. Rev. Lett. 22, 951-955. 
14. G. Mueller and J. W. Clark (1969) Status of Jastrow calculations of the $\Lambda$ binding energy of nuclear matter. Proc. International Conf. on Hypernuclear Physics, A. R. Bodmer and L. G. Hyman, eds. (Argonne National Laboratory, Argonne, IL), 598-618.

15. J. W. Clark and N.-C. Chao (1969) Effect of attractive nuclear forces on the onset of ferromagnetism in neutron star matter. Lett. Nuovo Cimento 2, 185-188.

16. J. W. Clark and G. Mueller (1969) Observations on $\Lambda$ overbinding in nuclear matter. Nuovo Cimento 64B, 217-239.

17. J. W. Clark (1969) Magnetic susceptibility of neutron matter. Phys. Rev. Lett. 23, $1463-1466$.

18. G. P. Mueller and J. W. Clark (1970) Ground state properties of alpha matter. Nucl. Phys. A155, 561-581.

19. J. W. Clark and M. L. Ristig (1970) Cluster expansion procedures for the correlated charge form factor. Nuovo Cimento A70, 313-322.

20. J. W. Clark and C.-H. Yang (1970) Superfluid ground state of neutron matter and other strongly-interacting many-fermion systems. Lett. Nuovo Cimento 3, $272-276 ; 2,379$.

21. B. Block, J. W. Clark, M. D. High, R. Malmin, and F. B. Malik (1971) Fission and the ion-ion interaction. Annals of Physics 62, 464-491.

22. M. L. Ristig, W. J. Ter Louw, and J. W. Clark (1971) Tensor correlations in nuclear matter. Phys. Rev. C 3, 1504-1513.

23. J. W. Clark (1971) Science fiction and the finitude of man. Washington University Magazine, Summer, 32-37.

24. C.-H. Yang and J. W. Clark (1971) Superfluid condensation energy of neutron matter. Nucl. Phys. A174, 49-62.

25. J. W. Clark, H. Heintzmann, W. Hillebrandt, and M. Grewing (1971) Nuclear forces, compressibility of neutron matter and the maximum mass of neutron stars. Astrophysical Lett. 10, 21-25.

26. N.-C. Chao, J. W. Clark, and C.-H. Yang (1972) Proton superfluidity in neutronstar matter. Nucl. Phys. A179, 320-332.

27. M. Miller, C. W. Woo, J. W. Clark, and W. J. Ter Louw (1972) Neutron-matter equations of state. Nucl. Phys. A184, 1-12.

28. M. L. Ristig, W. J. Ter Louw, and J. W. Clark (1972) Tensor correlations in nuclear matter: three-body effects. Phys. Rev. C 5, 695-706.

29. M. L. Ristig and J. W. Clark (1972) Role of the dispersion effect in the method of correlated basis functions. Phys. Rev. C 5, 1233-1237.

30. J. W. Clark and M. L. Ristig (1972) Subsidiary conditions on nuclear many-body theories. Phys. Rev. C 5, 1553-1561.

31. J. W. Clark and N.-C. Chao (1972) On the crystallization of neutronic matter. Nature Phys. Sci. 236, 37-38.

32. C.-H. Yang and J. W. Clark (1972) Thermodynamic critical field of superconducting neutron-star matter. Lett. Nuovo Cimento 4,

33. S.-O. Bäckman, J. W. Clark, W. J. Ter Louw, D. A. Chakkalakal, and M. L. Ristig (1972) Numerical comparison of reaction-matrix and Jastrow methods for nuclear matter: A reassessment. Phys. Lett. 41B, 247-250.

34. J. W. Clark and M. L. Ristig (1973) Embedding of the Brueckner approximation in the extended Jastrow scheme. Phys. Rev. C 7, 1792-1803. 
35. E. Krotscheck, J. Nitsch, M. L. Ristig, and J. W. Clark (1973) Grand-canonical formulation of renormalized cluster expansions for the nuclear-matter problem. Lett. Nuovo Cimento 6, 143-147.

36. E. Krotscheck, J. Nitsch, M. L. Ristig, and J. W. Clark (1973) A numerical study of renormalized Jastrow theory. Lett. Nuovo Cimento 6, 148-151.

37. M. L. Ristig and J. W. Clark (1973) The Iwamoto-Yamada cluster expansion: its structure and renormalization. Nucl. Phys. A199, 351-368.

38. J. W. Clark and M. L. Ristig (1973) The factor-cluster expansion as a framework for many-body theories. The Nuclear Many-Body Problem, F. Calogero and C. Ciofi degli Atti, eds. (Editrice Compositori, Bologna), Vol. II, 273-298.

39. M. L. Ristig and J. W. Clark (1973) Structure and renormalization of the factorcluster expansion. The Nuclear Many-Body Problem, F. Calogero and C. Ciofi degli Atti, eds. (Editrice Compositori, Bologna), Vol. II, 299-322.

40. J. W. Clark (1973) Solid neutron stars - fact or fiction? The Nuclear ManyBody Problem, F. Calogero and C. Ciofi degli Atti, eds. (Editrice Compositori, Bologna), Vol. II, 675-704.

41. J. W. Clark, N.-C. Chao, and C.-G. Källman (1973) Would alpha matter be solid? Physica Fennica 8, 335-344.

42. J. W. Clark (1974) The celestial timescope and the reincarnation of the universe. Washington University Magazine, Spring, 20-25.

43. J. W. Clark (1974) Inside a neutron star. Analog, September, 61-76.

44. J. W. Clark (1974) Review of A Random Walk in Science, J. Biol. Psych. 16, 45-46.

45. J. W. Clark and M. L. Ristig (1975) On short-range correlation effects in oneand two-nucleon ejection by light probe particles. Phys. Lett. 55B, 149-152.

46. J. W. Clark and D. G. Sandler (1975) Evidence against solidification of a model neutron system. Phys. Rev. D 11, 3365-3369.

47. N.-C. Chao and J. W. Clark (1975) Magnetic properties of neutron-star matter. Revista Brasileira de Fisica 5, 169-187.

48. M. L. Ristig and J. W. Clark (1975) A correlated model of the nuclear photoeffect. Nuovo Cimento 30A, 609-631.

49. J. W. Clark, P. M. Lam, and W. J. Ter Louw (1975) Perturbation corrections to the Jastrow energy for simple models of nuclear matter. Nucl. Phys. A255, $1-12$.

50. M. L. Ristig, P. M. Lam, and J. W. Clark (1975) Condensate fraction and momentum distribution of liquid helium. Phys. Lett. 55A, 101-103.

51. J. W. Clark, C.-G. Källman, C.-H. Yang, and D. A. Chakkalakal (1976) Effect of polarization on superfluidity in low-density neutron matter. Phys. Lett. 61B, 331-334.

52. M. L. Ristig and J. W. Clark (1976) Density matrix of quantum fluids. Phys. Rev. B 14, 2875-2887.

53. P. M. Lam, H. W. Jackson, M. L. Ristig, and J. W. Clark (1976) Dynamic structure function of liquid ${ }^{3} \mathrm{He}$. Phys. Lett. 58A, 454-456.

54. D. A. Chakkalakal, C.-H. Yang, and J. W. Clark (1976) Cluster-variational calculations for extended nuclear systems. Nucl. Phys. A271, 185-209.

55. P. M. Lam, J. W. Clark, and M. L. Ristig (1977) Density matrix and momentum distribution of helium liquids and nuclear matter. Phys. Rev. B 16; 222-230. 
56. J. W. Clark, M. T. Johnson, P. M. Lam, and J. G. Zabolitzky (1977) Numerical comparison of three theories of nuclear matter. Nucl. Phys. A283, 253-268.

57. M. L. Ristig, P. Hecking, P. M. Lam, and J. W. Clark (1977) Pairing energy of liquid ${ }^{4}$ He. Phys. Lett. 63A, 94-96.

58. K. E. Kürten, M. L. Ristig, and J. W. Clark (1977) Hypernetted-chain calculations for model nuclear matter. Lett. Nuovo Cimento 20, 313-318.

59. J. W. Clark, H. Rafelski, J. Rafelski, and J. Winston (1978) Quadrilogue on quarks. Washington University Magazine, Winter, 32-36.

60. J. W. Clark, P. M. Lam, J. G. Zabolitzky, and M. L. Ristig (1978) Theoretical momentum distributions for liquid ${ }^{3} \mathrm{He}$. Phys. Rev. B 17, 1147-1151.

61. K. E. Kürten, M. L. Ristig, and J. W. Clark (1978) Application of the method of correlated basis functions to nuclear matter. Phys. Lett. 74B, 153-157.

62. J. W. Clark and M. T. Johnson (1978) Alpha-cluster model of low-density nuclear matter. Clustering Aspects of Nuclear Structure and Nuclear Reactions, AIP Conf. Proc. 47, 544-545.

63. J. W. Clark and M. T. Johnson (1978) New microscopic calculations on alphaparticle matter. Proc. 2nd Latin American Workshop on Self-Consistent Theories of Condensed Matter, Trieste, 8-9.

64. J. W. Clark (1979) Variational theory of nuclear matter. Progress in Particle and Nuclear Physics 2, 89-199.

65. M. L. Ristig, K. E. Kürten, and J. W. Clark (1979) Density matrix and spindependent correlations in normal liquid ${ }^{3}$ He. Phys. Rev. B 19, 3539-3551.

66. D. G. Sandler and J. W. Clark (1979) A cluster-variational investigation of static density waves in nuclear matter. Nucl. Phys. A317, 23-36, Feenberg Memorial Issue.

67. K. E. Kürten, M. L. Ristig, and J. W. Clark (1979) A correlated-basis-functions approach to realistic nuclear matter. Nucl. Phys. A317, 87-115, Feenberg Memorial Issue.

68. V. C. Aguilera-Navarro, R. Barrera, J. W. Clark, M. de Llano, and A. Plastino (1979) Abnormal boson occupation in alpha matter. Phys. Lett. 80B, 327-331.

69. J. W. Clark, L. R. Mead, E. Krotscheck, K. E. Kürten, and M. L. Ristig (1979) Studies in the method of correlated basis functions I. A general survey. Nucl. Phys. A328, 45-72.

70. E. Krotscheck and J. W. Clark (1979) Studies in the method of correlated basis functions II. Graphical analysis and integral equation methods. Nucl. Phys. A328, 73-103.

71. J. W. Clark (1979) Update on the crisis in nuclear matter theory: A summary of the Trieste conference. Nucl. Phys. A328, 587-595.

72. J. W. Clark (1979) Survey of variational results for nuclear matter. Proc. 3rd Latin American Workshop on Self-Consistent Theories of Condensed Matter, Buenos Aires, 5-6.

73. E. Krotscheck and J. W. Clark (1980) Studies in the method of correlated basis functions III. Pair condensation in strongly-interacting Fermi systems. Nucl. Phys. A333, 77-115.

74. M. T. Johnson and J. W. Clark (1980) Variational theory of ideal alpha matter. KINAM, Revista de Fisica 2, 3-34. 
75. L. R. Mead and J. W. Clark (1980) Method of correlated basis functions for low levels of ${ }^{16} \mathrm{O}$. Phys. Lett. 90B, 331-334.

76. J. W. Clark (1980) Variational approaches to the nuclear-matter problem: A progress report. The Meson Theory of Nuclear Forces and Nuclear Matter, D. Schütte, K. Holinde, and K. Bleuler, eds. (B. I. Wissenschaftsverlag, Mannheim), 82-135.

77. T. J. Tarn, G. Huang, and J. W. Clark (1980) Modelling of quantum mechanical control systems. Mathematical Modelling 1, 109-121.

78. D. G. Sandler and J. W. Clark (1980) Electric-dipole sum rule in correlated nuclear matter. Nucl. Phys. A342, 213-228.

79. J. W. Clark, E. Krotscheck, and R. M. Panoff (1980) Ground-state properties of ${ }^{3} \mathrm{He} \uparrow$ and $\mathrm{D} \downarrow$ within the method of correlated basis functions. J. de Physique, Collog. 41, C7-197-212.

80. J. W. Clark (1980) Applications of the method of correlated basis functions to nuclear physics, astrophysics and low temperature physics. Proc. 4th PanAmerican Workshop on Self-Consistent Theories of Condensed Matter, Caracas.

81. D. G. Sandler and J. W. Clark (1981) Stability of nuclear matter against neutral pion condensation. Phys. Lett. 100B, 213-218.

82. J. W. Clark (1981) The correlated wave function approach to finite nuclear systems. The Many-Body Problem, Jastrow Correlations versus Brueckner Theory, R. Guardiola and J. Ros, eds. (Springer-Verlag, Berlin), 184-259.

83. E. Krotscheck and J. W. Clark (1981) Brueckner theory with Jastrow wave functions. The Many-Body Problem, Jastrow Correlations versus Brueckner Theory, R. Guardiola and J. Ros, eds. (Springer-Verlag, Berlin), 356-372.

84. J. W. Clark, D. G. Sandler, and E. Krotscheck (1981) Correlated RPA theory. Proc. 5th Pan-American Workshop on Condensed Matter Theories, Mexico City, 14-16.

85. D. G. Sandler, N.-H. Kwong, J. W. Clark, and E. Krotscheck (1981) New approaches to the study of collective excitations in strongly-interacting Fermi systems. Recent Progress in Many-Body Theories, J. G. Zabolitzky, M. de Llano, M. Fortes, and J. W. Clark, eds. (Springer-Verlag, Berlin), 228-234.

86. E. Krotscheck, R. A. Smith, and J. W. Clark (1981) Recent developments and future prospects in CBF theory. Recent Progress in Many-Body Theories, J. G. Zabolitzky, M. de Llano, M. Fortes, and J. W. Clark, eds. (Springer-Verlag, Berlin), 270-279.

87. E. Krotscheck, R. A. Smith, J. W. Clark, and R. M. Panoff (1981) Ground-state energetics of helium and deuterium fermion fluids. Phys. Rev. B 24, 6383-6403.

88. T. J. Tarn, J. W. Clark, C. K. Ong, and G. M. Huang (1982) Continuous-time quantum mechanical filter. Proc. Joint Workshop on Feedback and Synthesis of Linear and Nonlinear Systems, Bielefeld and Rome, D. Hinrichsen and A. Isidori, eds. (Springer-Verlag, Berlin).

89. V. C. Aguilera-Navarro, R. Barrera, M. de Llano, J. W. Clark, and A. Plastino (1982) Abnormal occupation in boson matter. Phys. Rev. C 25, 560-570

90. J. M. C. Chen, J. W. Clark, and D. G. Sandler (1982) An extension of RPA theory to strongly-interacting systems. Zeits. f. Physik A305, 223-229; 367.

91. R. M. Panoff, J. W. Clark, M. A. Lee, K. E. Schmidt, M. H. Kalos, and G. V. Chester (1982) Variational Monte Carlo calculations for spin-aligned deuterium. 
Phys. Rev. Lett. 48, 1675-1677.

92. J. W. Clark (1982) Variational methods and the effective interactions. Proceedings of the Third International Conference on Nuclear Reaction Mechanisms, Varenna, June 14-19, 1982, E. Gadioli, ed. (Ricerca Scientifica ed Educazione Permanente, Universita degli Studi di Milano), Supplemento n. 28, 464-479.

93. J. W. Clark (1982) Effective interactions for all seasons. Proc. 6th Pan-American Workshop on Condensed Matter Theories, St. Louis, 97-100.

94. Garng M. Huang, T. J. Tarn, and J. W. Clark (1983) On the controllability of quantum-mechanical systems. J. Math. Phys. 24, 2608-2618.

95. E. Krotscheck, J. W. Clark, and A. D. Jackson (1983) Properties of elementary excitations in spin-polarized liquid ${ }^{3} \mathrm{He}$. Phys. Rev. B 28, 5088-5099.

96. T. J. Tarn, J. W. Clark, and G. M. Huang (1984) Analytic controllability of quantum-mechanical systems. Proc. 1983 Symposium on the Mathematical Theory of Networks and Systems, P. A. Fuhrmann, ed. (Springer-Verlag, Berlin).

97. C. K. Ong, G. M. Huang, T. J. Tarn, and J. W. Clark (1984) Invertibility of quantum-mechanical control systems. Mathematical Systems Theory 17, 335350.

98. J. W. Clark, J. W. Chen, and G. H. Paine (1984) The brain as a many-body problem. Proc. 7th Pan American Workshop on Condensed Matter Theories, Altenberg.

99. J. W. Clark and E. Krotscheck (1984) Old dogs and new tricks: beyond the ground state with CBF theory. Recent Progress in Many-Body Theories, H. Kümmel and M. L. Ristig, eds. (Springer-Verlag, Berlin), 127-145.

100. J. W. Clark, J. V. Winston, and J. Rafelski (1984) Self-organization of neural networks. Phys. Lett. 102A, 207-211.

101. K. E. Kürten and J. W. Clark (1984) Variational Monte Carlo study of spindependent correlations in liquid ${ }^{3}$ He. Phys. Rev. B 30, 1342-1348.

102. J. W. Clark, E. Krotscheck, and B. Schwesinger (1984) Nuclear-matter particlehole force and a correlated RPA theory of ${ }^{16}$ O. Phys. Lett. 143B, 287-292.

103. M. F. Flynn, J. W. Clark, R. M. Panoff, O. Bohigas, and S. Stringari (1984) Models and approximations for the momentum distribution in nuclear matter. Nucl. Phys. A427, 253-277.

104. J. W. Clark, C. K. Ong, T. J. Tarn, and G. M. Huang (1985) Quantum nondemolition filters. Mathematical Systems Theory 18, 33-55.

105. J. W. Clark, E. Krotscheck, and B. Schwesinger (1985) Correlated RPA Treatment of Nuclei. Anales de Fisica 81 (Num. 1 Especial), 116-120.

106. M. F. Flynn, J. W. Clark, E. Krotscheck, R. A. Smith, and R. M. Panoff (1985) Ground-state properties of spin-aligned deuterium. Phys. Rev. B 32, 2945-2951.

107. K. E. Kürten and J. W. Clark (1985) Variational Monte Carlo study of heavyatom impurities in liquid ${ }^{4} \mathrm{He}$. Phys. Rev. $B$ 32, 2952-2959.

108. J. W. Clark, J. Rafelski, and J. V. Winston (1985) Brain without mind: Computer simulation of neural networks with modifiable neuronal interactions. Physics Reports 123(4), 215-273.

109. J. W. Clark, J. Cleymans, and J. Rafelski (1986) Clustered quark matter. Phys. Rev. C 33, 703-708.

110. K. E. Kürten and J. W. Clark (1986) Chaos in neural systems. Phys. Lett. 114A, 413-418. 
111. K. E. Kürten, M. L. Ristig, and J. W. Clark (1986) Variational theory of impurities in liquid ${ }^{4}$ He. Condensed Matter Theories, Vol. 1, F. B. Malik, ed. (Plenum, New York), pp. 107-114.

112. J. W. Clark, J. M. C. Chen, E. Krotscheck, and R. A. Smith (1986) Pairing in low-density neutron matter. Condensed Matter Theories, Vol. 1, F. B. Malik, ed. (Plenum, New York), pp. 313-326.

113. J. M. C. Chen, J. W. Clark, E. Krotscheck, and R. A. Smith (1986) Nucleonic superfluidity in neutron stars: ${ }^{1} \mathrm{~S}_{0}$ neutron pairing in the inner crust. Nucl. Phys. A451, 509-541.

114. J. W. Clark and T. J. Tarn (1987) Quantum nondemolition filtering. Information, Complexity, and Control in Quantum Physics, A. Blaquiere, S. Diner, and G. Lochak, eds. (Springer-Verlag, Berlin), pp. 331-346.

115. J. W. Clark (1987) Modeling of neural networks. Proceedings of the First Latin American School on Biophysics, ACIF Series - Volume 6, Bogota, Colombia, November 26 - December 8, 1984, R. Fayad, A. M. Rodrigues-Vargas, and G. Violini, eds. (World Scientific, Singapore), pp. 163-211.

116. K. E. Kürten and J. W. Clark (1987) Order and chaos in neural systems. Condensed Matter Theories, Vol. 2, P. Vashishta, R. K. Kalia, and R. F. Bishop, eds. (Plenum, New York), pp. 83-88.

117. E. Mavrommatis, R. Davé, and J. W. Clark (1987) The response function of the hard-sphere Fermi gas. Condensed Matter Theories, Vol. 2, P. Vashishta, R. K. Kalia, and R. F. Bishop, eds. (Plenum, New York), pp. 249-258.

118. R. M. Panoff and J. W. Clark (1987) Ground-state phases of polarized deuterium species. Phys. Rev. B 36, 5527-5539.

119. J. W. Clark (1988) Statistical mechanics of neural networks. Physics Reports 158, 91-158.

120. J. W. Clark, G. C. Littlewort, and J. Rafelski (1988) Topology, structure, and distance in quasirandom neural networks. Computer Simulation in Brain Science, R. J. M. Cotterill, ed. (Cambridge University Press, Cambridge), pp. 104118.

121. J. W. Clark, K. E. Kürten, and J. Rafelski (1988) Access and stability of cyclic modes in quasirandom networks of threshold neurons obeying a deterministic synchronous dynamics. Computer Simulation in Brain Science, R. J. M. Cotterill, ed. (Cambridge University Press, Cambridge), pp. 316-344.

122. G. C. Littlewort, J. W. Clark, and J. Rafelski (1988) Transition to cycling in neural networks. Computer Simulation in Brain Science, R. J. M. Cotterill, ed. (Cambridge University Press, Cambridge), pp. 345-356.

123. K. E. Kürten and J. W. Clark (1988) Exemplification of chaotic activity in nonlinear neural models obeying a deterministic dynamics in continuous time. Computer Simulation in Brain Science, R. J. M. Cotterill, ed. (Cambridge University Press, Cambridge), pp. 357-371.

124. J. W. Clark (1988) Probabilistic neural networks: In or out of equilibrium? Condensed Matter Theories, Vol. 3, J. S. Arponen, R. F. Bishop, and M. Manninen, eds. (Plenum, New York), pp. 381-391.

125. J. W. Clark (1988) Whither many-body theory? - A summary of the Oulu Conference. Recent Progress in Many-Body Theories, Vol. 1, A. J. Kallio, E. Pajanne, and R. F. Bishop, eds. (Plenum, New York), pp. 365-381. 
126. J. W. Chen, J. W. Clark, and K. E. Kürten (1988) Modelling of neural systems in continuous time. Mathematical and Computer Modelling 10, 503-513.

127. J. W. Clark and R. N. Silver (1988) Final-state effects in inclusive quasielastic electron scattering from nuclei: clues from quantum fluids. Proceedings of the Fifth International Conference on Nuclear Reaction Mechanisms, Varenna, June 13-18, 1988, E. Gadioli, ed. (Ricerca Scientifica ed Educazione Permanente, Universita degli Studi di Milano), Supplemento n. 66, pp. 531-540.

128. J. W. Clark (1988) Probabilistic neural networks. Evolution, Learning and Cognition, Y. C. Lee, ed. (World Scientific, Singapore), pp. 129-180.

129. T. J. Tarn, J. W. Clark, and G. M. Huang (1989) Local controllability of generalized quantum mechanical systems. Modeling and Control of Systems in Engineering, Quantum Mechanics, Economics and Biosciences, Proceedings of the Bellman Continuum 1988 Workshop, Sophia-Antipolis, France, A. Balquiere, ed. (Springer-Verlag, Berlin), pp. 161-172.

130. J. W. Clark (1989) Microscopic calculations on spin-polarized quantum fluids. Spin Polarized Quantum Systems, S. Stringari, ed. (World Scientific, Singapore), pp. 97-108.

131. J. W. Clark and R. M. Panoff (1989) Microscopic predictions for spin-aligned deuterium. Condensed Matter Theories, Vol. 4, J. Keller, ed. (Plenum, New York), pp. 1-15.

132. P. E. Sokol, R. N. Silver, and J. W. Clark (1989) Momentum distributions: an overview. Momentum Distributions, R. N. Silver and P. E. Sokol, eds. (Plenum, New York), pp. 1-35.

133. J. W. Clark and M. L. Ristig (1989) Overview of momentum distribution calculations. Momentum Distributions, R. N. Silver and P. E. Sokol, eds. (Plenum, New York), pp. 39-58.

134. M. L. Ristig and J. W. Clark (1989) Generalized momentum distributions. Momentum Distributions, R. N. Silver and P. E. Sokol, eds. (Plenum, New York), pp. 365-370.

135. M. L. Ristig and J. W. Clark (1989) Two-body density matrix of Bose fluids. Phys. Rev. B 40, 4355-4368.

136. J. W. Clark (1989) Introduction to neural networks. Non-linear Phenomena in Complex Systems, A. N. Proto, ed. (Elsevier, Amsterdam), pp. 1-102.

137. J. W. Clark, J.-W. Chen, and K. E. Kürten (1989) Analog simulation of circuits in the olfactory bulb. Models of Brain Function, R. J. M. Cotterill, ed. (Cambridge University Press, Cambridge), pp. 327-347.

138. R. D. Davé, J. W. Clark, and R. M. Panoff (1990) Elementary excitations of spin-aligned deuterium. Phys. Rev. B 41, 757-760.

139. J. C. Witt and J. W. Clark (1990) Experiments in artificial psychology: conditioning of asynchronous neural network models. Mathematical Biosciences 99, 77-104.

140. J. W. Clark and M. L. Ristig (1990) Generalized momentum distributions of quantum fluids. Condensed Matter Theories, Vol. 5, V. C. Aguilera-Navarro, ed. (Plenum, New York), pp. 47-60.

141. M. L. Ristig and J. W. Clark (1990) Two-body density matrix of a normal Fermi fluid. Phys. Rev. B 41, 8811-8823.

142. E. Mavrommatis and J. W. Clark (1990) Correlated RPA calculations for model 
nuclear matter. Condensed Matter Theories, Vol. 5, V. C. Aguilera-Navarro, ed. (Plenum, New York), pp. 97-107.

143. J. W. Clark (1990) Long-term behavior of neural networks. Relaxation in Complex Systems and Related Topics, I. A. Campbell and C. Giovannella, eds. (Plenum, New York), pp. 205-214.

144. M. L. Ristig and J. W. Clark (1990) Two-body density matrix of quantum fluids. Recent Progress in Many-Body Theories, Vol. 2, Y. Avishai, ed. (Plenum, New York), pp. 323-329.

145. M. P. Qian, G. Gong, and J. W. Clark (1991) Relative entropy and learning rules. Phys. Rev. A 43, 1061-1070.

146. A. Dabringhaus, M. L. Ristig, and J. W. Clark (1991) Vacuum ground and excited states of the U(1) lattice gauge Hamiltonian. Phys. Rev. D 43, 19781990.

147. J. W. Clark and S. Gazula (1991) Artificial neural networks that learn manybody physics. Condensed Matter Theories, Vol. 6, S. Fantoni and S. Rosati, eds. (Plenum, New York), pp. 1-24.

148. E. Mavrommatis and J. W. Clark (1991) Microscopic study of the response of nuclear matter. First Hellenic Symposium on Theoretical Nuclear Physics, G. Anagnostatos, M. Grypeos, E. Mavrommatis, D. Bonatsos, and L. Skouras, eds. (Aristotle University of Thessaloniki, Thessaloniki, Greece), pp. 11-26.

149. J. W. Clark (1991) Neural network modelling. Physics in Medicine and Biology 36, $1259-1317$.

150. J. W. Clark, R. D. Davé, and J. M. C. Chen (1992) Microscopic calculations of superfluid gaps. The Structure and Evolution of Neutron Stars, D. Pines, R. Tamagaki, and S. Tsuruta, eds. (Addison-Wesley, New York), pp. 134-147.

151. J. W. Clark, S. Gazula, and H. Bohr (1992) Teaching nuclear systematics to neural networks. Neural Networks: From Biology to High-Energy Physics, $\mathrm{O}$. Benhar, C. Bosio, P. del Giudice, and E. Tabet, eds. (ETS Editrice, Pisa, 1991), pp. 121-133.

152. J. W. Clark, S. Gazula, and H. Bohr (1992) Nuclear phenomenology with neural nets. Complex Dynamics in Neural Networks, J. G. Taylor, E. Caianiello, R. M. J. Cotterill, and J. W. Clark, eds. (Springer-Verlag, Berlin), pp. 305-322.

153. G. Senger, M. L. Ristig, C. E. Campbell, and J. W. Clark (1992) Correlated density-matrix theory of normal quantum fluids. Annals of Physics 218, 160196.

154. S. Gazula, J. W. Clark, and H. Bohr (1992) Learning and prediction of nuclear stability by neural networks. Nucl. Phys. A540, 1-26.

155. J. W. Clark, S. Gazula, K. A. Gernoth, J. Hasenbein, J. Prater, and H. Bohr (1992) Collective computation of many-body properties by neural networks. Recent Progress in Many-Body Theories, Vol. 3, T. L. Ainsworth, C. E. Campbell, B. E. Clements, and E. Krotscheck, eds. (Plenum, New York), pp. 371-386.

156. E. Mavrommatis, M. Petraki, J. W. Clark, and N. H. Kwong (1992) On a response function and two-body density matrix of nuclear matter. Second $\mathrm{Hel}$ lenic Symposium on Nuclear Physics, G. R. Anagnostatos, D. Bonatsos, and E. Mavrommatis, eds. (NCSR "Demokritos," Attiki, Greece, and the University of Athens, Panepistimiopolis, Kouponia, Greece), pp. 15-31.

157. J. W. Clark (1992) Views from the Ivory Tower's basement - A commentary 
on "The clock and the cloud: chaos and order in El diablo mundo." Revista de Estudios Hispánicos 26, 227-250.

158. J. W. Clark and K. A. Gernoth (1992) Teaching neural networks to do science. Structure: From Physics to General Systems, Vol. 2, M. Marinaro and G. Scarpetta, eds. (World Scientific, Singapore, 1992), pp. 64-77 [Eduardo R. Caianiello Commemorative Volumes].

159. K. A. Gernoth, J. W. Clark, J. S. Prater, and H. Bohr (1993) Neural network models of nuclear systematics. Phys. Lett. B300, 1-7.

160. J. W. Clark (1993) Neural network models: From biology to many-body phenomenology. Lecture notes for the Spring College on Many-Body Techniques, Isfahan University of Technology, Isfahan, Iran, April 20 - May 1, 1991, to be published by Plenum Press. [Also published in Proceedings of the International Nathiagali Summer College on Physics and Contemporary Needs, Vol. 17, K. Yaldram and M. M. Awais, eds. (Pinstech, Islamabad), pp. 15-102].

161. J. M. C. Chen, J. W. Clark, R. D. Davé, and V. V. Khodel (1993) Pairing gaps in nucleonic superfluids. Nucl. Phys. A555, 59-89 [Migdal Memorial Issue].

162. K. A. Gernoth, J. W. Clark, G. Senger, and M. L. Ristig (1993) Excitations of the surface of liquid ${ }^{4} \mathrm{He}$. Condensed Matter Theories, Vol. 8, L. Blum and F. B. Malik, eds. (Plenum, New York), pp. 195-203.

163. J. W. Clark, R. D. Davé, and J. M. C. Chen (1993) Nucleonic superfluids. Condensed Matter Theories, Vol. 8, L. Blum and F. B. Malik, eds. (Plenum, New York), pp. 227-242.

164. E. Mavrommatis, M. Petraki, and J. W. Clark (1993) Two-body density matrix of model nuclear matter. Third Hellenic Symposium on Nuclear Physics, R. Vlastou, C. T. Papadopoulos, and E. N. Gazis, eds. (NCSR “Demokritos," Atti$\mathrm{ki}$, Greece, and the University of Athens, Panepistimiopolis, Kouponia, Greece), pp. 88-103.

165. J. W. Clark, E. Mavrommatis, and M. Petraki (1993) Generalized momentum distribution of nuclear matter. Acta Physica Polonica B 24, 659-684 [Janusz Dabrowski Festschrift Issue].

166. K. A. Gernoth, J. W. Clark, G. Senger, and M. L. Ristig (1994) Surface modes of liquid ${ }^{4} \mathrm{He}$. Phys. Rev. B 49, 15836-15848.

167. J. W. Clark, K. A. Gernoth, and M. L. Ristig (1994) Connectionist many-body phenomenology. Condensed Matter Theories, Vol. 9, J. W. Clark, A. Sadiq, and K. A. Shoaib, eds. (Nova Science Publishers, Commack, NY), pp. 519-537.

168. K. A. Gernoth and J. W. Clark (1994) Resonant vapor modes in ${ }^{4} \mathrm{He}$ vaporliquid systems. Condensed Matter Theories, Vol. 9, J. W. Clark, A. Sadiq, and K. A. Shoaib, eds. (Nova Science Publishers, Commack, NY), pp. 37-53.

169. K. A. Gernoth and J. W. Clark (1994) Resonant states of the ${ }^{4}$ He liquid-vapor interface. J. Low Temp. Phys. 96, 153-175.

170. K. A. Gernoth, J. W. Clark, and M. L. Ristig (1995) The surface of liquid ${ }^{4} \mathrm{He}$ at nonzero temperatures. Zeits. f. Physik B98, 337-340.

171. K. A. Gernoth and J. W. Clark (1995) Neural networks that learn to predict probabilities: Global models of nuclear stability and decay. Neural Networks 8 , 291-311.

172. K. A. Gernoth, J. W. Clark, and M. L. Ristig (1995) Correlated density matrix theory of spatially inhomogeneous Bose fluids. Condensed Matter Theories, 
Vol. 10, M. Casas, M. de Llano, J. Navarro, and A. Polls, eds. (Nova Science Publishers, Commack, NY), in press.

173. J. W. Clark and K. A. Gernoth (1995) Statistical modeling of nuclear masses with neural network algorithms. Condensed Matter Theories, Vol. 10, M. Casas, M. de Llano, J. Navarro, and A. Polls, eds. (Nova Science Publishers, Commack, $\mathrm{NY}$ ), in press.

174. M. L. Ristig, G. Senger, M. Serhan, and J. W. Clark (1995) Correlated density matrix theory of boson superfluids. Annals of Physics 243, 247-279.

175. K. A. Gernoth and J. W. Clark (1995) A modified backpropagation algorithm for training neural networks on data with error bars. Computer Physics Communications 88, 1-22.

176. J. W. Clark, K. A. Gernoth, and M. L. Ristig (1995) Higher-order probabilistic perceptrons as Bayesian inference engines. Preprint.

177. J. W. Clark (1995) Pathways to the quantum realm - Presentation of the fifth Eugene Feenberg Memorial Medal in Many-Body Physics to David M. Ceperley. Recent Progress in Many-Body Theories, Vol. 4, E. Schachinger, H. Mitter, and H. Sormann, eds. (Plenum, New York), pp. 449-454.

178. J. W. Clark, K. A. Gernoth, and M. L. Ristig (1995) Connectionist statistical inference. Recent Progress in Many-Body Theories, Vol. 4, E. Schachinger, H. Mitter, and H. Sormann, eds. (Plenum, New York), pp. 283-292.

179. E. Mavrommatis, M. Petraki, and J. W. Clark (1995) Fermi hypernetted chain evaluation of a generalized momentum distribution for model nuclear matter. Phys. Rev. C 51, 1849-1858.

180. E. Mavrommatis, M. Petraki, and J. W. Clark (1995) Study of the generalized momentum distribution of model nuclear matter. Advances in Nuclear Physics, Proceedings of the Fifth Symposium of Nuclear Physics, Hellenic Nuclear Physics Society, C. Syros and C. Ronchi, eds. (European Commission, Karlsruhe, Luxembourg, Patras, 1995), pp. 139-157.

181. K. A. Gernoth and J. W. Clark (1995) Neural network models of nuclear and noisy data. Proceedings of the Fourth International Workshop on Software Engineering and Artificial Intelligence for High Energy and Nuclear Physics, B. Denby, ed. (World Scientific, Singapore), in press.

182. V. A. Khodel, J. W. Clark, and V. R. Shaginyan (1995) Rearrangement of the electron Fermi surface in layered compounds. Solid State Communications $\mathbf{9 6 ,}$ 353-357.

183. J. W. Clark (1996) Control of quantum many-body dynamics: Designing quantum scissors. Condensed Matter Theories, Vol. 11, E. V. Ludeña, ed. (Nova Science Publishers, Commack, NY).

184. V. A. Khodel, V. V. Khodel, and J. W. Clark (1996) Solution of the gap equation in neutron matter. Nucl. Phys. A, in press.

185. M. L. Ristig, J. W. Kim, and J. W. Clark (1996) The Z(2) lattice gauge vacuum: A correlated basis functions analysis. Phys. Rev. D, submitted.

\section{MONOGRAPHS - REVIEWS}

J. W. Clark (1979) Variational Theory of Nuclear Matter. In Progress in Particle and Nuclear Physics, D. H. Wilkinson, ed. (Pergamon, Oxford, 1979), Vol. 2. 
J. W. Clark, J. Rafelski, and J. V. Winston (1985) Brain Without Mind: Computer Simulation of Neural Networks with Modifiable Neuronal Interactions. Physics Reports 123(4), 215-273.

J. W. Clark (1988) Statistical Mechanics of Neural Networks. Physics Reports 158, 91-158.

J. W. Clark (1989) Introduction to Neural Networks. In Nonlinear Phenomena in Complex Systems, A. N. Proto, ed. (Elsevier, Amsterdam, 1989).

J. W. Clark (1991) Neural network modelling. Physics in Medicine and Biology 36, $1259-1317$.

\section{BOOKS AND PROCEEDINGS EDITED}

Recent Progress in Many-Body Theories, J. G. Zabolitzky, M. de Llano, M. Fortes, and J. W. Clark (Springer-Verlag, Berlin, 1981).

Methods for the Many-Body Problem, J. M. C. Chen, J. W. Clark, and P. Suntharothok-Priesmeyer (Washington University, St. Louis, 1983).

Complex Dynamics in Neural Networks, J. G. Taylor, E. Caianiello, R. M. J. Cotterill, and J. W. Clark, eds. (Perspectives in Neural Computing Series, SpringerVerlag, Berlin, 1992).

Condensed Matter Theories, Vol. 9, J. W. Clark, A. Sadiq, and K. A. Shoaib, eds. (Nova Science Publishers, Commack, NY, 1994). 